\title{
Article \\ Coincidence Theory in the Coercive Case and Minimax Inequalities
}

\author{
Donal O'Regan
}

Citation: O'Regan, D. Coincidence Theory in the Coercive Case and Minimax Inequalities. Symmetry 2021, 13, 1220. https://doi.org/10.3390/ sym 13071220

Academic Editors: Aviv Gibali, Ioan Rașa and Sergei D. Odintsov

Received: 15 April 2021

Accepted: 1 July 2021

Published: 7 July 2021

Publisher's Note: MDPI stays neutral with regard to jurisdictional claims in published maps and institutional affiliations.

Copyright: (c) 2021 by the authors. Licensee MDPI, Basel, Switzerland. This article is an open access article distributed under the terms and conditions of the Creative Commons Attribution (CC BY) license (https:// creativecommons.org/licenses/by/ $4.0 /)$.
School of Mathematics, Statistics and Applied Mathematics, National University of Ireland, H91TK33 Galway, Ireland; donal.oregan@nuigalway.ie

Abstract: We established coincidence results between maps with continuous selections and admissible maps. Both the compact and coercive cases were considered, and our argument relied on new coincidence ideas established recently by the author. Using our coincidence theory, we established new analytic alternatives, which then generate new minimax inequalities of the Neumann-Sion type.

Keywords: continuous selections; admissible maps; coincidence theory; analytic alternatives; minimax inequalities

\section{Introduction and Coincidence Results in the Compact Case}

For multimaps $T, H: X \rightarrow 2^{Y}$, the coincidence problem considers putting conditions on the spaces $X$ and $Y$ and the maps $T$ and $H$, so that there exists an $x \in X$ with $T(x) \cap$ $H(x) \neq \varnothing$. Coincidence results generate minimax inequalities, which have applications in game theory and abstract economies (see [1]). For an introduction to coincidence theory and applications, we refer the reader to the book of Gorniewicz [2]. In this paper, we established coincidence results between multivalued maps with continuous selections and maps in the Park and Gorniewicz class in both the compact and coercive cases. Our arguments presented here to guarantee a coincidence point are based on a fixed-point theorem in the literature [3]. Using our coincidence theorems, we present a variety of analytic alternatives [4-6], which then generate some minimax inequalities of the NeumannSion type [5-7]. This paper can be viewed as a companion paper to [8]; in our paper, here, the maps were in very general classes of maps (namely the maps of Park), and in addition, we considered a new case, namely the coercive case, which was not discussed in [8]. We note that in many biological and physical models, asymmetry plays an important role, and general coincidence point theory enables one to consider symmetry and asymmetry in one general setting.

Let $H$ be the Čech homology functor with compact carriers and coefficients in the field of rational numbers $K$ from the category of Hausdorff topological spaces and continuous maps to the category of graded vector spaces and linear maps of degree zero. Thus, $H(X)=\left\{H_{q}(X)\right\}$ (here, $X$ is a Hausdorff topological space) is a graded vector space, $H_{q}(X)$ being the $q$-dimensional Čech homology group with compact carriers of $X$. For a continuous map $f: X \rightarrow X, H(f)$ is the induced linear map $f_{\star}=\left\{f_{\star q}\right\}$ where $f_{\star q}: H_{q}(X) \rightarrow H_{q}(X)$. A space $X$ is acyclic if $X$ is nonempty, $H_{q}(X)=0$ for every $q \geq 1$, and $H_{0}(X) \approx K$.

Let $X, Y$, and $\Gamma$ be Hausdorff topological spaces. A continuous single-valued map $p: \Gamma \rightarrow X$ is called a Vietoris map (written $p: \Gamma \Rightarrow X$ ) if the following two conditions are satisfied:

(i) for each $x \in X$, the set $p^{-1}(x)$ is acyclic;

(ii) $p$ is a perfect map, i.e., $p$ is closed, and for every $x \in X$, the set $p^{-1}(x)$ is nonempty and compact. 
Let $\phi: X \rightarrow Y$ be a multivalued map (note that, for each $x \in X$, we assume $\phi(x)$ is a nonempty subset of $Y$ ). A pair $(p, q)$ of single-valued continuous maps of the form $X \stackrel{p}{\leftarrow} \Gamma \stackrel{q}{\rightarrow} Y$ is called a selected pair of $\phi$ (written $(p, q) \subset \phi)$ if the following two conditions hold:

(i) $p$ is a Vietoris map;

(ii) $q\left(p^{-1}(x)\right) \subset \phi(x)$ for any $x \in X$.

Next, we considered the admissible maps of Gorniewicz [2]. An upper semicontinuous map $\phi: X \rightarrow Y$ with compact values is said to be admissible (and we write $\phi \in \operatorname{Ad}(X, Y)$ ) provided there exists a selected pair $(p, q)$ of $\phi$. An example of an admissible map is a Kakutani map. An upper semicontinuous map $\phi: X \rightarrow K(Y)$ is said to Kakutani (and we write $\phi \in K a k(X, Y)$ ); here, $K(Y)$ denotes the family of nonempty, convex, compact subsets of $Y$.

Let $Z$ and $W$ be subsets of Hausdorff topological vector spaces $Y_{1}$ and $Y_{2}$ and $G$ a multifunction. We say $G \in D K T(Z, W)$ [1,9] (due to Ding, Kim, and Tan) if $W$ is convex; there exists a map $S: Z \rightarrow W$ with $c o(S(x)) \subseteq G(x)$ for $x \in Z$ and $S(x) \neq \varnothing$ for each $x \in Z$, and $S^{-1}(w)=\{z \in Z: w \in S(z)\}$ is open (in $Z$ ) for each $w \in W$.

In [8], we established the following coincidence results.

Theorem 1. Let $X$ and $Y$ be subsets of a Hausdorff topological vector space $E$ with $X$ convex. Suppose $F \in \operatorname{Ad}(X, Y)$ is a compact map and $G \in D K T(Y, X)$. Then, there exists an $x \in X$ with $G^{-1}(x) \cap F(x) \neq \varnothing$.

Theorem 2. Let $X$ and $Y$ be convex subsets of a Hausdorff topological vector space E. Suppose $F \in D K T(X, Y)$ is a compact map and $G \in D K T(Y, X)$. Then, there exists an $x \in X$ with $G^{-1}(x) \cap F(x) \neq \varnothing$.

In fact, Theorem 1 can be extended to a more general class of maps, namely the $P K$ maps of Park. Let $X$ and $Y$ be Hausdorff topological spaces. Given a class $\mathcal{X}$ of maps, $\mathcal{X}(X, Y)$ denotes the set of maps $F: X \rightarrow 2^{Y}$ (nonempty subsets of $Y$ ) belonging to $\mathcal{X}$ and $\mathcal{X}_{c}$ the set of finite compositions of maps in $\mathcal{X}$. We let:

$$
\mathcal{F}(\mathcal{X})=\{Z: \text { Fix } F \neq \varnothing \text { for all } F \in \mathcal{X}(Z, Z)\}
$$

where Fix F denotes the set of fixed points of $F$.

The class $\mathcal{U}$ of maps is defined by the following properties:

(i) $\mathcal{U}$ contains the class $\mathrm{C}$ of single-valued continuous functions;

(ii) each $F \in \mathcal{U}_{c}$ is upper semicontinuous and compact valued;

(iii) $B^{n} \in \mathcal{F}\left(\mathcal{U}_{c}\right)$ for all $n \in\{1,2, \ldots$.$\} ; here, B^{n}=\left\{x \in \mathbf{R}^{n}:\|x\| \leq 1\right\}$.

We say $F \in P K(X, Y)$ if for any compact subset $K$ of $X$ there is a $G \in \mathcal{U}_{c}(K, Y)$ with $G(x) \subseteq F(x)$ for each $x \in K$. Recall that $P K$ is closed under compositions.

A nonempty subset $C$ of a topological vector space $E$ is said to be admissible if, for every compact subset $K$ of $C$ and every neighborhood $V$ of the origin zero of $E$, there exists a continuous map $h: K \rightarrow C$ such that $x-h(x) \in V$ for all $x \in K$ and $h(K)$ is contained in a finite-dimensional subspace of $E$. Recall that every nonempty convex subset of a locally convex topological vector space is admissible. The following result was established in [3].

Theorem 3. Let $X$ be an admissible convex subset of a Hausdorff topological vector space E. Then, any compact $F \in P K(X, X)$ has a fixed point.

In fact, the result in Theorem 3 can be improved (see $[10,11])$. We present one such result below. By a space, we mean a Hausdorff topological space. Let $Q$ be a class of topological spaces. A space $Y$ is an extension space for $Q$ (written $Y \in E S(Q)$ if for all 
$X \in Q$ and all $K \subseteq X$ closed in $X$, any continuous function $f_{0}: K \rightarrow Y$ extends to a continuous function $f: X \rightarrow Y$.

Theorem 4. Let $X \in E S$ (compact) and $\Psi \in P K(X, X)$ be a compact map. Then, there exists an $x \in X$ with $x \in \Psi(x)$.

We are now in a position to extend Theorem 1.

Theorem 5. Let $X$ and $Y$ be subsets of a Hausdorff topological vector space $E$ with $X$ convex. Suppose $F \in P K(X, Y)$ is a compact map and $G \in D K T(Y, X)$. Then, there exists an $x \in X$ with $G^{-1}(x) \cap F(x) \neq \varnothing$.

Proof. Let $K$ be a compact subset of $Y$ with $F(X) \subseteq K$. Since $G \in D K T(Y, X)$, then $G \in$ $D K T(K, X)$. To establish this first note, there exists a map $S: Y \rightarrow X$ with $c o(S(y)) \subseteq G(y)$ for $y \in Y, S(y) \neq \varnothing$ for each $y \in Y$, and $S^{-1}(x)$ is open (in $Y$ ) for each $x \in X$. Let $S^{\star}$ denote the restriction of $S$ to $K$, and note that if $x \in X$, then (note that $K \subseteq Y$ ):

$$
\left(S^{\star}\right)^{-1}(x)=\left\{z \in K: x \in S^{\star}(z)\right\}=\{z \in K: x \in S(z)\}=K \cap\{z \in Y: x \in S(z)\}
$$

which is open in $K \cap Y=K$. Thus, $G \in D K T(K, X)$, and from [1,9], there exists a selection $g \in C(K, X)$ (the space of continuous single-valued maps $\theta: K \rightarrow X)$ of $G$ and a finite subset $A$ of $X$ with $g(K) \subseteq c o(A)$. Note that $g F \in P K(c o(A), c o(A))$ and $c o(A)$ is a compact convex subset in a finite-dimensional subspace of $E$, so there exists an $x \in X$ with $x \in g(F(x))$.

Theorem 6. Let $X$ and $Y$ be subsets of a Hausdorff topological vector space $E$ with $X$ convex and $Y$ paracompact. Suppose $G \in D K T(Y, X)$ is a compact map and $F \in P K(X, Y)$. In addition, assume $X \in E S\left(\right.$ compact). Then, there exists an $x \in X$ with $G^{-1}(x) \cap F(x) \neq \varnothing$.

Proof. Since $Y$ is paracompact, then from [1,9], there exists a selection $g \in C(Y, X)$ of G. Now, $g F \in P K(X, X)$ is a compact map, so Theorem 4 guarantees an $x \in X$ with $x \in g F(x)$.

Remark 1. In Theorem 6, if we replace $X \in E S$ (compact) with (i) $Y \in E S$ (compact) and (ii) $F$ is upper semicontinuous and compact valued, then the result in Theorem 6 is again true. The proof is immediate once we note that $F g \in P K(Y, Y)$ is a compact map, so Theorem 4 guarantees that there exists a $y \in Y$ with $y \in F g(y)$.

Theorem 7. Let $X$ and $Y$ be subsets of a Hausdorff topological vector space $E$ with $X$ convex and $Y$ closed. Suppose $F \in P K(X, Y)$ is upper semicontinuous and compact valued and $G \in D K T(Y, X)$ is a compact map. Let $K$ be the compact subset of $X$ with $G(Y) \subseteq K$; let $L(K)$ be the linear span of $K$ (i.e., the smallest linear subspace of $E$ that contains $K$ ); assume $F(L(K)) \subseteq L(K)$ and $Y \cap L(K) \in E S($ compact $)$. Then, there exists a $y \in Y$ with $G(y) \cap F^{-1}(y) \neq \varnothing$.

Proof. Let $K$ be a compact set with $G(Y) \subseteq K$, and let $L(K)$ be the linear span of $K$ as described above. We now show $G \in D K T(Y \cap L(K), L(K))$. To establish this first note, since $G \in D K T(Y, X)$, there exists a map $S: Y \rightarrow X$ with co $(S(y)) \subseteq G(y)$ for $y \in Y$, $S(y) \neq \varnothing$ for each $y \in Y$, and $S^{-1}(x)=\{z \in Y: x \in S(z)\}$ is open (in $Y$ ) for each $x \in X$. Let $S^{\star}$ denote the restriction of $S$ to $Y \cap L(K)$. Note that $c o\left(S^{\star}(y)\right) \subseteq G(y)$ for $y \in Y \cap L(K)$, $S^{\star}(y) \neq \varnothing$ for each $y \in Y \cap L(K)$, and if $x \in L(K)$, then:

$$
\begin{aligned}
\left(S^{\star}\right)^{-1}(x) & =\left\{z \in Y \cap L(K): x \in S^{\star}(z)\right\}=\{z \in Y \cap L(K): x \in S(z)\} \\
& =L(K) \cap\{z \in Y: x \in S(z)\}=L(K) \cap S^{-1}(y)
\end{aligned}
$$


which is open in $Y \cap L(K)$. Thus, $G \in D K T(Y \cap L(K), L(K))$. Now, recall that $L(K)$ is Lindelöf, so paracompact [12], and since $Y \cap L(K)$ is closed in $L(K)$, then $Y \cap L(K)$ is paracompact. Thus, there exists a selection $g \in C(Y \cap L(K), L(K))$ of $G$. Notice that since $F(L(K)) \subseteq L(K), F g \in P K(Y \cap L(K), Y \cap L(K))$ is a compact map. Now, Theorem 4 guarantees a $y \in Y \cap L(K)$ with $y \in F g(y))$.

\section{Coincidence Results in the Coercive Case}

Let $Z$ and $W$ be subsets of Hausdorff topological vector spaces. A multivalued map $Q: Z \rightarrow W$ is said to be coercive if there exists a compact subset $K$ of $Z$ and a compact convex subset $C$ of $W$ such that $Q(x) \cap C \neq \varnothing$ for $x \in Z \backslash K$. We now also consider a subclass of the $D K T(Z, W)$ maps (see [5]). Let $G$ be a multifunction, and we say $G \in \Phi^{\star}(Z, W)[5]$ if $W$ is convex and there exists a map $S: Z \rightarrow W$ with $S(x) \subseteq G(x)$ for $x \in Z, S(x) \neq \varnothing$ and has convex values for each $x \in Z$, and $S^{-1}(w)$ is open (in $Z$ ) for each $w \in W$.

Theorem 8. Let $X$ and $Y$ be subsets of a Hausdorff topological vector space $E$ with $X$ convex and $F \in A d(X, Y)$. Let $G \in \Phi^{\star}(Y, X)$, and suppose there exists a coercive map $S: Y \rightarrow X$ with $S(y) \subseteq G(y)$ for $y \in Y, S(y) \neq \varnothing$ and has convex values for each $y \in Y$, and $S^{-1}(x)$ is open (in $Y$ ) for each $x \in X$. Then, there exists an $x \in X$ with $G^{-1}(x) \cap F(x) \neq \varnothing$.

Proof. Let $K \subseteq Y$ be compact, $C \subseteq X$ be convex and compact, and $S(y) \cap C \neq \varnothing$ for $y \in Y \backslash K$. Now, since $G \in \Phi^{\star}(Y, X)$, then $G \in \Phi^{\star}(K, X)$, since with $S^{\star}$ denoting the restriction of $S$ to $K$, note that if $x \in X$, then (note that $K \subseteq Y$ ):

$$
\left(S^{\star}\right)^{-1}(x)=\left\{z \in K: x \in S^{\star}(z)\right\}=\{z \in K: x \in S(z)\}=K \cap\{z \in Y: x \in S(z)\}
$$

which is open in $K \cap Y=K$. Thus, $G \in \Phi^{\star}(K, X)$ (with $K$ compact), so from [5,9], there exists a selection $g \in C(K, X)$ of $G$ (in fact, we have (see [5,9]) $g(x) \subseteq S(x) \subseteq G(x)$ for $x \in K)$ and a finite subset $B$ of $X$ with $g(K) \subseteq c o(B)$. Let:

$$
C_{0}=\operatorname{co}(\operatorname{co}(B) \cup C)
$$

which is a convex compact [13] (p. 125) subset of $X$. Note that $F \in A d\left(C_{0}, Y\right)$ is a compact map. Let $G^{\star}(y)=G(y) \cap C_{0}$ for $y \in Y$, and we claim $G^{\star} \in \Phi^{\star}\left(Y, C_{0}\right)$. Let $S^{\star \star}(y)=$ $S(y) \cap C_{0}$ for $y \in Y$. If $y \in Y \backslash K$, then $S^{\star \star}(y)=S(y) \cap C_{0} \neq \varnothing$, since $S(y) \cap C \neq \varnothing$ and $C \subseteq C_{0}$, whereas if $y \in K$, then since $g(K) \subseteq S(K)$ and $g(K) \subseteq \operatorname{co}(B) \subseteq C_{0}$, we have $S^{\star \star}(y)=S(y) \cap C_{0} \neq \varnothing$. Next, if $y \in Y$, then $S^{\star \star}(y)=S(y) \cap C_{0} \subseteq G(y) \cap C_{0}=G^{\star}(y)$. Finally, note that if $x \in C_{0}$, then:

$$
\left(S^{\star \star}\right)^{-1}(x)=\left\{y \in Y: x \in S^{\star \star}(y)\right\}=\left\{y \in Y: x \in S(y) \cap C_{0}\right\}=\{y \in Y: x \in S(y)\}
$$

which is open in $Y$. Thus, $G^{\star} \in \Phi^{\star}\left(Y, C_{0}\right)$ (in fact, also a compact map). Now, Theorem 1 (with $F$ and $G^{\star}$ ) guarantees that there exists an $x \in C_{0}$ with $F(x) \cap\left(G^{\star}\right)^{-1}(x) \neq \varnothing$, i.e., there exists a $y$ with $y \in F(x)$ and $y \in\left(G^{\star}\right)^{-1}(x)$ (i.e., $\left.x \in G^{\star}(y)=G(y) \cap C_{0}\right)$. Thus, $G^{-1}(x) \cap F(x) \neq \varnothing$.

Corollary 1. Let $X$ be a convex subset of a Hausdorff topological vector space $E$. Let $G \in$ $\Phi^{\star}(X, X)$, and suppose there exists a coercive map $S: X \rightarrow X$ with $S(y) \subseteq G(y)$ for $y \in X$, $S(y) \neq \varnothing$ and has convex values for each $y \in X$, and $S^{-1}(x)$ is open (in X) for each $x \in X$. Then, there exists an $x \in X$ with $x \in G^{-1}(x)$.

We next replace $A d$ maps in Theorem 8 with the $P K$ maps with an extra assumption.

Theorem 9. Let $X$ and $Y$ be subsets of a Hausdorff topological vector space $E$ with $X$ convex, $F \in P K(X, Y)$, and $F$ is upper semicontinuous with compact values. Let $G \in \Phi^{\star}(Y, X)$, and suppose there exists a coercive map $S: Y \rightarrow X$ with $S(y) \subseteq G(y)$ for $y \in Y, S(y) \neq \varnothing$ for 
each $y \in Y$, and $S^{-1}(x)$ is open (in $Y$ ) for each $x \in X$. Then, there exists an $x \in X$ with $G^{-1}(x) \cap F(x) \neq \varnothing$.

Proof. Let $K, C, g, C_{0}$ and $G^{\star}$ be as in Theorem 8. Furthermore, note that since $C_{0}$ is compact and $F$ is upper semicontinuous with compact values, $F \in P K\left(C_{0}, Y\right)$ is a compact map. The argument in Theorem 8 guarantees that $G^{\star} \in \Phi^{\star}\left(Y, C_{0}\right)$. Now, apply Theorem 5 (with $F$ and $G^{\star}$ ).

Remark 2. Note that $G^{\star} \in \Phi^{\star}\left(Y, C_{0}\right)$ in Theorem 9 is a compact map, so we could remove the assumption that $F$ is upper semicontinuous with compact values in the statement of Theorem 9 and use Theorem 6 instead of Theorem 5 in the proof, provided extra assumptions are added.

Theorem 10. Let $X$ and $Y$ be convex subsets of a Hausdorff topological vector space $E$ with $F \in D K T(X, Y)$. Let $G \in \Phi^{\star}(Y, X)$, and suppose there exists a coercive map $S: Y \rightarrow X$ with $S(y) \subseteq G(y)$ for $y \in Y, S(y) \neq \varnothing$ and has convex values for each $y \in Y$, and $S^{-1}(x)$ is open (in $Y)$ for each $x \in X$. Then, there exists an $x \in X$ with $G^{-1}(x) \cap F(x) \neq \varnothing$.

Proof. Let $K, C, g, C_{0}$, and $G^{\star}$ be as in Theorem 8 . The argument in Theorem 8 guarantees that $G^{\star} \in \Phi^{\star}\left(Y, C_{0}\right)$ is a compact map. Next, we show $F \in D K T\left(C_{0}, Y\right)$. To see this, note that there exists a map $M: X \rightarrow Y$ with $c o(M(x)) \subseteq F(x)$ for $x \in X, M(x) \neq \varnothing$ for each $x \in X$, and $M^{-1}(y)$ is open (in $X$ ) for each $y \in Y$. Let $M^{\star}$ denote the restriction of $S$ to $C_{0}$, and note that if $y \in Y$, then (note that $C_{0} \subseteq X$ ):

$$
\left(M^{\star}\right)^{-1}(y)=\left\{z \in C_{0}: y \in M^{\star}(z)\right\}=\left\{z \in C_{0}: y \in M(z)\right\}=C_{0} \cap\{z \in X: y \in M(z)\}
$$

which is open in $C_{0} \cap X=C_{0}$. Thus, $F \in D K T\left(C_{0}, Y\right)$. Now, Theorem 2 (with $G^{\star}$, the compact map, and $F)$ guarantees that there exists a $y \in Y$ with $F^{-1}(y) \cap G^{\star}(y) \neq \varnothing$, so there exists an $x$ with $x \in F^{-1}(y)$ (i.e., $\left.y \in F(x)\right)$ and $x \in G^{\star}(y)=G(y) \cap C_{0}$ (i.e., $x \in G(y)$, so $\left.y \in G^{-1}(x)\right)$. Thus, $G^{-1}(x) \cap F(x) \neq \varnothing$.

Next, we present some new analytic alternatives from our coincidence results (these analytic alternatives then generate some minimax inequalities). A coincidence theorem produces an analytic alternative, and in this paper, to illustrate the strategy involved, we generate analytic alternatives off Theorem 2, Corollary 1, and Theorems 9 and 10.

Theorem 11. Let $X$ and $Y$ be convex subsets of a Hausdorff topological vector space E. Let $f, t, s, g: X \times Y \rightarrow \mathbf{R}$ with $g(x, y) \leq s(x, y) \leq t(x, y) \leq f(x, y)$ for all $(x, y) \in X \times Y$. Fix $\lambda \in \mathbf{R}$, and let:

$$
F(x)=\{y \in Y: f(x, y)<\lambda\}, M(x)=\{y \in Y: t(x, y)<\lambda\} \text { for } x \in X
$$

and:

$$
G(y)=\{x \in X: g(x, y)>\lambda\}, H(y)=\{x \in X: s(x, y)>\lambda\} \text { for } y \in Y \text {. }
$$

Assume co $(F(x)) \subseteq M(x)$ for each $x \in X, F^{-1}(y)$ is open (in $\left.X\right)$ for each $y \in Y$, and $M: X \rightarrow Y$ is a compact map. Furthermore, suppose co $(G(y)) \subseteq H(y)$ for each $y \in Y$ and $G^{-1}(x)$ is open (in $Y$ ) for each $x \in X$. Then, at least one of the following is true:

(1) There exists $x_{0} \in X$ with $f\left(x_{0}, y\right) \geq \lambda$ for all $y \in Y$;

(2) There exists $y_{0} \in Y$ with $g\left(x, y_{0}\right) \leq \lambda$ for all $x \in X$.

Proof. Note that $F(x) \neq \varnothing$ for every $x \in X$ holds or does not hold. Similarly, $G(y) \neq \varnothing$ for every $y \in Y$ holds or does not hold.

Consider first the case when $F(x) \neq \varnothing$ for every $x \in X$ and $G(y) \neq \varnothing$ for every $y \in Y$. Then (note that $F$ is a selection of $M$ and $G$ is a selection of $H), M \in D K T(X, Y)$ (is a compact map) and $H \in D K T(Y, X)$. Now, Theorem 2 (with $M$ and $H$ ) guarantees an 
$x_{0} \in X$ with $H^{-1}\left(x_{0}\right) \cap M\left(x_{0}\right) \neq \varnothing$. Let $y_{0} \in H^{-1}\left(x_{0}\right) \cap M\left(x_{0}\right)$, then, since $y_{0} \in M\left(x_{0}\right)$, we have $t\left(x_{0}, y_{0}\right)<\lambda$, and since $y_{0} \in H^{-1}\left(x_{0}\right)$, we have $s\left(x_{0}, y_{0}\right)>\lambda$. Thus, $t\left(x_{0}, y_{0}\right)<$ $\lambda<s\left(x_{0}, y_{0}\right)$, a contradiction.

Consider next the case when $F(x) \neq \varnothing$ for every $x \in X$ does not hold. Then, there exists an $x_{0} \in X$ with $F\left(x_{0}\right)=\varnothing$, i.e., $f\left(x_{0}, y\right) \geq \lambda$ for all $y \in Y$.

Finally, consider the case when $G(y) \neq \varnothing$ for every $y \in Y$ does not hold. Then, there exists $y_{0} \in Y$ with $G\left(y_{0}\right)=\varnothing$, i.e., $g\left(x, y_{0}\right) \leq \lambda$ for all $x \in X$.

Theorem 12. Let $X$ and $Y$ be convex subsets of a Hausdorff topological vector space E. Let $f, t, s, g: X \times Y \rightarrow \mathbf{R}$ with $g(x, y) \leq s(x, y) \leq t(x, y) \leq f(x, y)$ for all $(x, y) \in X \times Y$. Fix $\lambda \in \mathbf{R}$, and let:

$$
F(x)=\{y \in Y: f(x, y)<\lambda\}, M(x)=\{y \in Y: t(x, y)<\lambda\} \text { for } x \in X
$$

and:

$$
G(y)=\{x \in X: g(x, y)>\lambda\}, H(y)=\{x \in X: s(x, y)>\lambda\} \text { for } y \in Y .
$$

Assume co $(F(x)) \subseteq M(x)$ for each $x \in X$ and $F^{-1}(y)$ is open (in $X$ ) for each $y \in Y$. Suppose there is a $K \subseteq Y$ compact and $C \subseteq X$ convex and compact with $G(y) \cap C \neq \varnothing$ for $y \in Y \backslash K$. Assume $G^{-1}(x)$ is open (in $Y$ ) for each $x \in X$ and $G(y)$ is convex-valued for each $y \in Y$. Then, at least one of the following is true:

(1) There exists $x_{0} \in X$ with $f\left(x_{0}, y\right) \geq \lambda$ for all $y \in Y$;

(2) There exists $y_{0} \in K$ with $g\left(x, y_{0}\right) \leq \lambda$ for all $x \in X$.

Proof. Note that $F(x) \neq \varnothing$ for every $x \in X$ holds or does not hold. Similarly, $G(y) \neq \varnothing$ for every $y \in K$ holds or does not hold.

Consider first the case when $F(x) \neq \varnothing$ for every $x \in X$ and $G(y) \neq \varnothing$ for every $y \in K$. Then, $M \in D K T(X, Y)$ (note that $F$ is a selection of $M$ ) and $H \in \Phi^{\star}(Y, X)$ (note that the coercive map $G: Y \rightarrow X$ is a selection of $H$ ). Now, Theorem 10 (with $M$ and $H$ ) guarantees an $x_{0} \in X$ with $H^{-1}\left(x_{0}\right) \cap M\left(x_{0}\right) \neq \varnothing$. Let $y_{0} \in H^{-1}\left(x_{0}\right) \cap M\left(x_{0}\right)$, and as in Theorem 11, we have $t\left(x_{0}, y_{0}\right)<\lambda<s\left(x_{0}, y_{0}\right)$, a contradiction.

Consider next the case when $F(x) \neq \varnothing$ for every $x \in X$ does not hold. Then, there exists an $x_{0} \in X$ with $F\left(x_{0}\right)=\varnothing$, i.e., $f\left(x_{0}, y\right) \geq \lambda$ for all $y \in Y$.

Finally, consider the case when $G(y) \neq \varnothing$ for every $y \in K$ does not hold. Then, there exists $y_{0} \in K$ with $G\left(y_{0}\right)=\varnothing$, i.e., $g\left(x, y_{0}\right) \leq \lambda$ for all $x \in X$.

Theorem 13. Let $X$ be a convex subset of a Hausdorff topological vector space E. Let $f, g$ : $X \times X \rightarrow \mathbf{R}$ with $g(x, y) \leq f(x, y)$ for all $(x, y) \in X \times X$. Fix $\lambda \in \mathbf{R}$, and let:

$$
F(y)=\{x \in X: f(x, y)>\lambda\} \text { and } G(y)=\{x \in X: g(x, y)>\lambda\} \text { for } y \in X .
$$

Assume there is a $K \subseteq X$ compact and $C \subseteq X$ convex and compact with $G(y) \cap C \neq \varnothing$ for $y \in X \backslash K$. Suppose $G^{-1}(x)$ is open (in $\left.X\right)$ for each $x \in X$ and $G(y)$ is convex-valued for each $y \in X$. Then, either:

(1) There exists $y_{0} \in X$ with $f\left(y_{0}, y_{0}\right)>\lambda$;

or

(2) There exists $y_{0} \in K$ with $g\left(x, y_{0}\right) \leq \lambda$ for all $x \in X$. occurs.

Proof. Note $G(y) \neq \varnothing$ for every $y \in K$ holds or does not hold.

Consider first the case when $G(y) \neq \varnothing$ for every $y \in K$. Then, $F \in \Phi^{\star}(X, X)$ (note that the coercive map $G: X \rightarrow X$ is a selection of $F)$, so Corollary 1 guarantees a $y_{0} \in X$ with $y_{0} \in F\left(y_{0}\right)$, i.e., $f\left(y_{0}, y_{0}\right)>\lambda$. 
Finally, consider the case when $G(y) \neq \varnothing$ for every $y \in K$ does not hold. Then, there exists $y_{0} \in K$ with $G\left(y_{0}\right)=\varnothing$, i.e., $g\left(x, y_{0}\right) \leq \lambda$ for all $x \in X$.

Finally, we use Theorem 9 to generate the following analytic alternative. Note one could also generate analytic alternatives from Theorems 5-7; we leave the obvious details to the reader.

Theorem 14. Let $X$ and $Y$ be subsets of a Hausdorff topological vector space $E$ with $X$ convex. Let $s, g: X \times Y \rightarrow \mathbf{R}$ with $g(x, y) \leq s(x, y)$ for all $(x, y) \in X \times Y$. Fix $\lambda \in \mathbf{R}$, and let:

$$
G(y)=\{x \in X: g(x, y)>\lambda\} \text { and } H(y)=\{x \in X: s(x, y)>\lambda\} \text { for } y \in Y .
$$

Suppose $F \in P K(X, Y)$ and $F$ is upper semicontinuous with compact values. Assume there is a $K \subseteq Y$ compact and $C \subseteq X$ convex and compact with $G(y) \cap C \neq \varnothing$ for $y \in Y \backslash K$. Assume $G^{-1}(x)$ is open (in $Y$ ) for each $x \in X$ and $G(y)$ is convex-valued for each $y \in Y$. Then, either:

(1) There exists $x_{0} \in X$ and $y_{0} \in F\left(x_{0}\right)$ with $s\left(x_{0}, y_{0}\right)>\lambda$;

or

(2) There exists $y_{0} \in K$ with $g\left(x, y_{0}\right) \leq \lambda$ for all $x \in X$. occurs.

Proof. Note that $G(y) \neq \varnothing$ for every $y \in K$ holds or does not hold.

Consider first the case when $G(y) \neq \varnothing$ for every $y \in K$. Then, $H \in \Phi^{\star}(Y, X)$ (note that the coercive map $G: Y \rightarrow X$ is a selection of $H$ ), so Theorem 9 guarantees that there exists $x_{0} \in X$ with $H^{-1}\left(x_{0}\right) \cap F\left(x_{0}\right) \neq \varnothing$. Let $y_{0} \in H^{-1}\left(x_{0}\right) \cap F\left(x_{0}\right)$. Then, $y_{0} \in F\left(x_{0}\right)$, and since, $y_{0} \in H^{-1}\left(x_{0}\right)$, we have $s\left(x_{0}, y_{0}\right)>\lambda$.

Finally, consider the case when $G(y) \neq \varnothing$ for every $y \in K$ does not hold. Then, there exists $y_{0} \in K$ with $G\left(y_{0}\right)=\varnothing$ i.e., $g\left(x, y_{0}\right) \leq \lambda$ for all $x \in X$.

We now use Theorems 11 and 12 to generate minimax inequalities of the NeumannSion type.

Theorem 15. Let $X$ and $Y$ be convex subsets of a Hausdorff topological vector space E. Let $f, t, s, g: X \times Y \rightarrow \mathbf{R}$ with $g(x, y) \leq s(x, y) \leq t(x, y) \leq f(x, y)$ for all $(x, y) \in X \times Y$. For each $\lambda \in \mathbf{R}$, let:

$$
F_{\lambda}(x)=\{y \in Y: f(x, y)<\lambda\}, M_{\lambda}(x)=\{y \in Y: t(x, y)<\lambda\} \text { for } x \in X
$$

and:

$$
G_{\lambda}(y)=\{x \in X: g(x, y)>\lambda\}, H_{\lambda}(y)=\{x \in X: s(x, y)>\lambda\} \text { for } y \in Y .
$$

Assume co $\left(F_{\lambda}(x)\right) \subseteq M_{\lambda}(x)$ for each $x \in X, F_{\lambda}^{-1}(y)$ is open (in $\left.X\right)$ for each $y \in Y$, and $M_{\lambda}: X \rightarrow Y$ is a compact map. Furthermore, suppose co $\left(G_{\lambda}(y)\right) \subseteq H_{\lambda}(y)$ for each $y \in Y$ and $G_{\lambda}^{-1}(x)$ is open (in $Y$ ) for each $x \in X$. Then:

$$
\alpha \equiv \inf _{y \in Y} \sup _{x \in X} g(x, y) \leq \sup _{x \in X} \inf _{y \in Y} f(x, y) \equiv \beta .
$$

Proof. Let $\beta<\infty$ and $\alpha>-\infty$ (otherwise, we are finished). Suppose $\beta<\alpha$. Then, there exists a $\lambda$ with:

$$
\beta<\lambda<\alpha \text {. }
$$

We now apply Theorem 11. If there exists a $y_{0} \in Y$ with $g\left(x, y_{0}\right) \leq \lambda$ for all $x \in X$, then $\alpha=\inf _{y \in Y} \sup _{x \in X} g(x, y) \leq \lambda$, and this contradicts (2.1). If there exists an $x_{0} \in X$ with 
$f\left(x_{0}, y\right) \geq \lambda$ for all $y \in Y$, then $\beta=\sup _{x \in X} \inf _{y \in Y} f(x, y) \geq \lambda$, and this contradicts (2.1). As a result, we have $\alpha \leq \beta$.

Theorem 16. Let $X$ and $Y$ be convex subsets of a Hausdorff topological vector space E. Let $f, t, s, g: X \times Y \rightarrow \mathbf{R}$ with $g(x, y) \leq s(x, y) \leq t(x, y) \leq f(x, y)$ for all $(x, y) \in X \times Y$. For each $\lambda \in \mathbf{R}$, let:

$$
F_{\lambda}(x)=\{y \in Y: f(x, y)<\lambda\}, M_{\lambda}(x)=\{y \in Y: t(x, y)<\lambda\} \text { for } x \in X
$$

and:

$$
G_{\lambda}(y)=\{x \in X: g(x, y)>\lambda\}, H_{\lambda}(y)=\{x \in X: s(x, y)>\lambda\} \text { for } y \in Y .
$$

Assume co $\left(F_{\lambda}(x)\right) \subseteq M_{\lambda}(x)$ for each $x \in X$ and $F_{\lambda}^{-1}(y)$ is open (in $\left.X\right)$ for each $y \in Y$. Suppose there is a $K_{\lambda} \subseteq Y$ compact and $C_{\lambda} \subseteq X$ convex and compact with $G_{\lambda}(y) \cap C_{\lambda} \neq \varnothing$ for $y \in Y \backslash K_{\lambda}$. Assume $G_{\lambda}^{-1}(x)$ is open (in $Y$ ) for each $x \in X$ and $G_{\lambda}(y)$ is convex-valued for each $y \in Y$. Then:

$$
\alpha \equiv \inf _{y \in Y} \sup _{x \in X} g(x, y) \leq \sup _{x \in X} \inf _{y \in Y} f(x, y) \equiv \beta .
$$

Proof. Let $\beta<\infty$ and $\alpha>-\infty$ (otherwise, we are finished). Suppose $\beta<\alpha$. Then, there exists a $\lambda$ with $\beta<\lambda<\alpha$. We now apply Theorem 12. If there exists an $x_{0} \in X$ with $f\left(x_{0}, y\right) \geq$ $\lambda$ for all $y \in Y$, then $\inf _{y \in Y} f\left(x_{0}, y\right) \geq \lambda$, so $\beta=\sup _{x \in X} \inf _{y \in Y} f(x, y) \geq \lambda$, a contradiction. If there exists a $y_{0} \in K_{\lambda}$ with $g\left(x, y_{0}\right) \leq \lambda$ for all $x \in X$, then $\sup _{x \in X} g\left(x, y_{0}\right) \leq \lambda$, so $\alpha=\inf _{y \in Y} \sup _{x \in X} g(x, y) \leq \lambda$, a contradiction. Thus, (see Theorem 12) $\alpha \leq \beta$.

We now use Theorem 14 to generate a minimax inequality.

Theorem 17. Let $X$ and $Y$ be subsets of a Hausdorff topological vector space $E$ with $X$ convex. Let $s, g: X \times Y \rightarrow \mathbf{R}$ with $g(x, y) \leq s(x, y)$ for all $(x, y) \in X \times Y$. For each $\lambda \in \mathbf{R}$, let:

$$
G_{\lambda}(y)=\{x \in X: g(x, y)>\lambda\} \text { and } H_{\lambda}(y)=\{x \in X: s(x, y)>\lambda\} \text { for } y \in Y .
$$

Suppose $F \in P K(X, Y)$ and $F$ is upper semicontinuous with compact values. Assume there is a $K_{\lambda} \subseteq Y$ compact and $C_{\lambda} \subseteq X$ convex and compact with $G_{\lambda}(y) \cap C_{\lambda} \neq \varnothing$ for $y \in Y \backslash K_{\lambda}$. Assume $G_{\lambda}^{-1}(x)$ is open (in $Y$ ) for each $x \in X$ and $G_{\lambda}(y)$ is convex-valued for each $y \in Y$. Then:

$$
\inf _{y \in Y} \sup _{x \in X} g(x, y) \leq \sup \{s(x, y): x \in X, y \in Y, y \in F(x)\} \equiv \gamma .
$$

Proof. Let $\gamma<\infty$ (otherwise, we are finished). We now apply Theorem 14 with $\lambda=\gamma$. Note that from the definition of $\gamma$, (1) in Theorem 14 (with $\lambda=\gamma$ ) cannot occur. Thus, there exists $y_{0} \in K_{\gamma}$ with $g\left(x, y_{0}\right) \leq \gamma$ for all $x \in X$, so $\sup _{x \in X} g\left(x, y_{0}\right) \leq \gamma$. Thus, $\inf _{y \in Y} \sup _{x \in X} g(x, y) \leq \gamma$.

Remark 3. In the statement of Theorem 17, one could replace "For each $\lambda \in \mathbf{R}$, let" with "For $\lambda=\gamma$, let".

\section{Conclusions}

In this paper, we presented coincidence results for multivalued maps for the compact and coercive cases. Our coincidence theory produced analytic alternatives, which generated minimax inequalities.

Author Contributions: Conceptualization, D.O.; methodology, D.O.; validation, D.O.; formal analysis, D.O.; writing — original draft preparation, D.O.; funding acquisition, D.O. The author read and agreed the published version of the manuscript.

Funding: This research received no external funding. 
Institutional Review Board Statement: Not applicable.

Informed Consent Statement: Not applicable.

Data Availability Statement: Not applicable.

Conflicts of Interest: The author declares no conflict of interest.

\section{References}

1. Lim, L.J.; Park, S.; Yu, Z.T. Remarks on fixed points, maximal elements and equilibria of generalized games. J. Math. Anal. Appl. 1999, 233, 581-596.

2. Gorniewicz, L. Topological Fixed Point Theory of Multivalued Mappings; Kluwer Acad. Publishers: Dordrecht, The Netherlands, 1991.

3. Park, S. A unified fixed point theory for multimaps on topological vector spaces. J. Korean Math. Soc. 1988, 35, 803-829.

4. Agarwal, R.P.; O'Regan, D. Coincidence theory for $U_{c}^{\kappa}$ maps and inequalities. J. Nonlinear Convex Anal. 2004, 5, $265-274$.

5. Ben-El-Mechaiekh, H.; Deguire, P.; Granas, A. Points fixes et coincidences pour les applications multivoques II (Applications de type $\Phi$ and $\left.\Phi^{\star}\right)$. C. R. Acad. Sci. 1982, 295, 381-384.

6. O'Regan, D. A general coincidence theory for set valued maps. Zeitschrift Anal. Anwendungen 1999, 18, 701-712. [CrossRef]

7. O'Regan, D. Coincidence theory for CS maps with applications. Commun. Appl. Anal. 1999, 3, 433-446.

8. O'Regan, D. Coincidence results and Leray-Schauder alternatives between multivalued maps with continuous selections and admissible maps. Topol. Appl. 2020, 284, 107368. [CrossRef]

9. Ding, X.P.; Kim, W.K.; Tan, K.K. A selection theorem and its applications. Bull. Aust. Math. Soc. 1992, 46, 205-212. [CrossRef]

10. Agarwal, R.P.; O'Regan, D.; Park, S. Fixed point theory for multimaps in extension type spaces. J. Korean Math. Soc. 2002, 39, 579-591. [CrossRef]

11. O'Regan, D. Fixed point theory for extension type spaces and essential maps on topological spaces. Fixed Point Theory Appl. 2004, 1, 13-20. [CrossRef]

12. Fournier, G.; Granas, A. The Lefschetz fixed point theorem for some classes of non-metrizable spaces. J. Math. Pures Appl. 1973, 52, 271-283.

13. Aliprantis, C.D.; Border, K.C. Infinite Dimensional Analysis; Springer: Berlin, Germany, 1994. 